\title{
Geomagnetic effect of the Bering Sea meteoroid
}

\author{
B. G. Gavrilov ${ }^{1}$, V. A. Pilipenko ${ }^{2,3}$, Y. V. Poklad ${ }^{1}$, and I. A. Ryakhovsky ${ }^{1}$ \\ Received 07 August 2020; accepted 06 November 2020; published 24 November 2020.
}

Possibilities of studies of the geomagnetic effects produced by the interaction of a cosmic bodies with the magnetosphere-ionosphere-atmosphere system are very limited due to extremely small number of examined events. Here we present geomagnetic observations at an array of magnetometers during Bering Sea Bolide event on December 18, 2018 when a space body entered the Earth's atmosphere and exploded at the altitude of $\sim 25 \mathrm{~km}$ near Kamchatka. It has been found that the short-lived electromagnetic signal appeared before the explosion and, consequently, was trigged by the passage of a meteoroid through the inner magnetosphere. Geomagnetic disturbances of the same duration and frequency of oscillations were detected both in the area adjacent to the explosion site in the Northern hemisphere and in the magnetically conjugated area in the Southern hemisphere. These observations may be provisionally interpreted as a triggered excitation of resonant field line oscillations in the inner magnetosphere by the fast-moving meteoroid. The magnetosphere is often in a metastable state, when even a weak external trigger can stimulate an internal instability and wave generation. The appearance of a diamagnetic effect during partial ablation of a meteoroid could cause a local disturbance of the geomagnetic field and its propagation in the magnetic force tube. KEYWORDS: meteoroid; geomagnetic response; geomagnetic pulsations; ionosphere; magnetosphere.

Citation: Gavrilov, B. G., V. A. Pilipenko, Y. V. Poklad, and I. A. Ryakhovsky (2020), Geomagnetic effect of the Bering Sea meteoroid, Russ. J. Earth. Sci., 20, ES6009, doi:10.2205/2020ES000748.

\section{Keypoints}

1. On December 18, 2018 the Bering Sea Bolide entered the Earth's atmosphere vertically and exploded at altitude of $\sim 25 \mathrm{~km}$ with a probable yield $4-35 \mathrm{kT}$.

2. Geomagnetic oscillatory response with frequencies $25-35 \mathrm{mHz}$ was recorded $\sim 3-10 \mathrm{~min}$ before the bolide explosion, during its passage though the inner magnetosphere.

\footnotetext{
${ }^{1}$ Sadovsky Institute of Geosphere Dynamics, Russian Academy of Sciences (IDG RAS), Moscow, Russia

${ }^{2}$ Schmidt Institute of Physics of the Earth, Russian Academy of Sciences (IPE RAS), Moscow, Russia

${ }^{3}$ Geophysical Center RAS (GC RAS), Moscow, Russia
}

Copyright 2020 by the Geophysical Center RAS. http://rjes.wdcb.ru/doi/2020ES000748-res.html
3. The geomagnetic response is probably due to trigger excitation of resonant field line oscillations.

\section{Introduction}

An interaction of conductive body with plasma is accompanied by excitation of various electromagnetic disturbances and emissions. In the terrestrial ionosphere these effects are manifested as generation of geomagnetic impulses and noise by take-offs of rockets [Chernogor, 2013], plasma injection in rocket experiments [Gavrilov et al., 2003, movement of large-scale space systems (e.g., tether, Shuttle orbiter, International Space Station, etc.) in the upper ionosphere [Dobrowolny and Veltri, 1986. This class of phenomena comprises the geo- 
magnetic effects caused by the fall of large cosmic bodies, meteor showers, and bolides [Savchenko, 1975, 1976.

Studies of geomagnetic disturbances associated with the fall of meteoroids, fireballs, and meteor showers have been conducted for several decades [Bronshten, 1991]; Kalashnikov, 1949, 1952] was the first who reported the observation of weak changes of the geomagnetic field attributed to meteors. However, many subsequent studies gave no evidence of a magnetic effect due to a single meteor [Hawkins, 1958]. Observations with a high sensitivity magnetometer concluded that most individual meteors do not have any associated geomagnetic pulsation activity, but some of the larger meteors do produce magnetic effects [Ellyett and Fraser, 1963].

The greatest number of studies is devoted, perhaps, to the geomagnetic effect of the Tunguska phenomenon [Ivanov and Medvedev, 1965; Nemchinov et al., 1999. Although this event has been known for a long time, the responsible physical mechanism has not been firmly established [Bronstein, 2002 Ivanov, 1967. Geomagnetic fluctuations with quasi-period 5-13 min were observed during the Chelyabinsk meteoroid event, but $\sim 45$ min before the explosion [Chernogor, 2018]. Rare information on geomagnetic effects of cosmic objects hints that transients or pulsations of geomagnetic field can be generated by relatively small cosmic bodies (even with a diameter of the order of 1 $\mathrm{m})$ and propagate over distances of several thousand kilometers [Chernogor, 2018]. Bodies with such dimensions invade the atmosphere quite often - once per week or month, whereas a space object commensurate with the Tunguska body falls to the Earth on average once every 100-200 years [Brown et al., 2002]. Because the impact of bolides and meteoroids on the near-Earth environment is a rare and unpredictable event, the observational information is very limited.

A large class of ionospheric and geomagnetic perturbations associated with meteor/bolides is caused by perturbation of the ionosphere by an acoustic wave coming from the region of the main energy release caused by the object destruction. These effects are physically similar to electromagnetic/ionospheric effects associated with strong ground or atmospheric explosions [Zetser et al., 2004]. Owing to waveguide effects in the atmosphere the acoustic waves from explosion can propagate to distances about several thousand $\mathrm{km}$ [Adushkin et al., 2004]. Acoustic waves can modulate the plasma density and electric current in the conductive E-layer of the ionosphere, thus causing a geomagnetic response on the ground.

Besides that, other mechanisms of magnetic effect produced by bolides/meteoroids are feasible. For example, the interaction of the geomagnetic field with the plasma formed in the head of the meteoroid may be significant for geomagnetic response [Bronstein, 2002]. However, in-depth studies of these effects are very limited owing to an extremely small number of examined events.

On December 18, 2018 a space body entered the Earth's atmosphere and exploded at the altitude of $\sim 25 \mathrm{~km}$. Later it became known as Kamchatka meteor or Bering Sea Bolide. The explosion, which was estimated to be $\sim 10$ times more powerful than the atomic bomb explosion in Hiroshima $(\sim 21 \mathrm{kT})$, was not seen by anyone. It was discovered only as a result of post-processing of photos taken by NASA satellites.

The purpose of our work is to examine possible electromagnetic effects, which may be caused by interaction of a cosmic body with the magnetosphereionosphere-atmosphere system. In search for possible effects, we consider not only the final explosion and acoustic blast, but disturbances during passage of the bolide through the inner magnetosphere and ionosphere.

\section{The Bering Sea Event}

According to NASA fireball database https: //cneos.jpl.nasa.gov/fireballs the explosion occurred on December 18, 2018 at 23:48:20 UT in the point with geographic coordinates $56.9^{\circ} \mathrm{N}$ and $172.4^{\circ} \mathrm{E}$. The radiative energy was estimated to be $31 \mathrm{kT}$ and the total energy was about $173 \mathrm{kT}$. According to this estimate, the Bering Sea Meteoroid (BSM) explosion is the third in power after the Tunguska event $(\sim 20000 \mathrm{kT})$ and the Chelyabinsk meteoroid $(\sim 440 \mathrm{kT})$ [Popova et al., 2013, but much more powerful than the Vitim meteoroid $(\sim 2 \mathrm{kT})$. The BSM was estimated to have a core diameter $\sim 10$ meters and weighed $\sim 1360$ tons.

NASA experts estimated the speed of BSM en- 


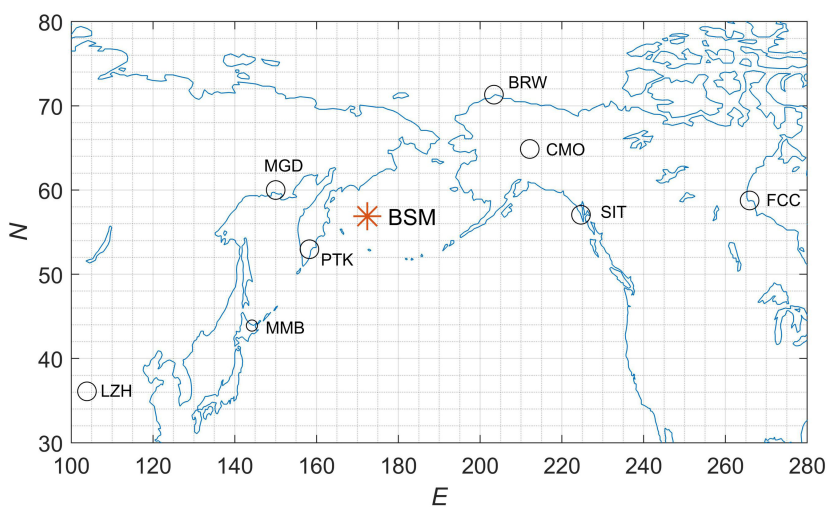

Figure 1. The location of the BSM explosion and the most important geomagnetic stations in the area of the explosion.

try of $\sim 32 \mathrm{~km} / \mathrm{s}$, with the speed components $V x=6.3, V y=-3$ and $V z=-31.2 \mathrm{~km} / \mathrm{s}$. Thus, the BSM entered the atmosphere almost vertically. Therefore, the BSM entered the inner magnetosphere/plasmasphere $\left(\sim 3-4 R_{E}\right)$ about 10-13 minutes before the explosion.

In addition to optical data, acoustic observations at the international monitoring system (IMS) are available. IMS operates in compliance with the comprehensive nuclear-test-ban treaty (CTBT) and includes $\sim 60$ infrasound stations throughout the globe. Infrasound response to the BSM explosion was identified at distances from $\sim 1000$ to $\sim 8000 \mathrm{~km}$ at 13 stations located at azimuths from $17^{\circ}$ to $349^{\circ}$ [Negraru and Johnson, 2019]. The period of registered acoustic signals was in the range from 2.4 to 20.1 seconds. The equivalent energy release for the bolide may be estimated from the relationship $\log Y=3.34 \log \tau-2.58$, where $Y$ is the yield (in $\mathrm{kT}$ ) and $\tau$ is the dominant signal period (in sec) [Revelle, 1997]. As a result, the bolide energy was estimated to be between 4 and $35 \mathrm{kT}$. This estimate is significantly lower than those obtained from the optical data. The same conclusion was reached by Gordeev et al. 2019 based on Russian infrasound measurements in Kamchatka. According to their data, the TNT equivalent of the bolide is estimated to be even lower, about 1.3-8.2 $\mathrm{kT}$. Hence, the explosion power of $173 \mathrm{kT}$ derived from the NASA optical observations seems to be overestimated, since in this case the period of infrasound oscillations should have been at least $30 \mathrm{~s}$ [Negraru and Johnson, 2019].

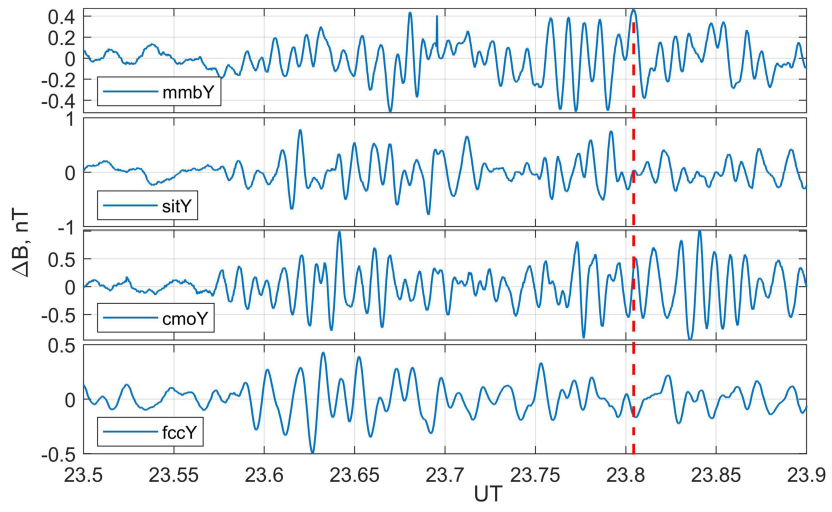

Figure 2. Variations of $\mathrm{Y}$ components of the magnetic field according to data of the Memambetsu (MMB), Sitka (SIT), College (CMO) and Fort Churchill (FCC) observatories on December 18, 2018. The red dashed line shows the moment of the explosion.

\section{Geomagnetic Observations}

To detect and investigate possible disturbances of the geomagnetic field, we have selected fluxgate magnetometer data from available INTERMAGNET stations with 1-sec cadence that are located in the vicinity of the explosion site in the Northern hemisphere and its conjugate point in the Southern hemisphere. Besides fluxgate magnetometers from the INTERMAGNET array, we examine the data from more sensitive search-coil magnetometers at Magadan (MGD) and Paratunka (PET) stations with $64 \mathrm{~Hz}$ sampling frequency deployed at subauroral latitudes within the framework of the PWING project [Shiokawa et al., 2017]. Figure 1] shows the location of the explosion and position of the most important stations, whereas Table 1 provides information on coordinates of the magnetic observatories considered and their distances $R$ from the explosion site. All stations are in the Sun-illuminated or twilight zone during the event https://www. timeanddate.com/worldclock/sunearth.html|.

Figure 2 shows variations of the magnetic field at several magnetic stations in the region of BSM explosion: Memambetsu (MMB), Sitka (SIT), College (CMO), and Fort Churchill (FCC). The geomagnetic effect of the explosion itself and any aftereffects were not detected. About 12 minutes before the explosion (since 23:36 UT) periodic oscillations emerge that last for $\sim 15 \mathrm{~min}$. The largest signal peak-to-peak amplitude $\sim 1 \mathrm{nT}$ is observed in 
Table 1. Magnetic stations whose data is used in the work

\begin{tabular}{|c|c|c|c|c|}
\hline $\begin{array}{l}\text { IAGA } \\
\text { code }\end{array}$ & Name & $\begin{array}{l}\text { Lat. } \\
\text { N }\left(^{\circ}\right)\end{array}$ & $\begin{array}{l}\text { Long. } \\
\text { E }\left(^{\circ}\right)\end{array}$ & $\begin{array}{c}\text { Distance } \\
\text { to ex- } \\
\text { plosion } \\
\text { R (km) }\end{array}$ \\
\hline ASP & Alice Springs & -23.77 & 133.88 & 9640 \\
\hline BRW & Barrow & 71.32 & 203.38 & 2160 \\
\hline CKI & $\begin{array}{l}\text { Cocos-Keeling } \\
\text { Islands }\end{array}$ & -12.188 & 96.834 & 10275 \\
\hline $\mathrm{CMO}$ & College & 64.87 & 212.14 & 2290 \\
\hline $\mathrm{CNB}$ & Canberra & -35.32 & 149.36 & 10450 \\
\hline EYR & Eyrewell & -43.474 & 172.39 & 10130 \\
\hline FCC & Fort Churchill & 58.759 & 265.91 & 5096 \\
\hline $\mathrm{MCQ}$ & $\begin{array}{l}\text { Macquarie } \\
\text { Island }\end{array}$ & -54.5 & 159.9 & 12425 \\
\hline MGD & Magadan & 60 & 150 & 1300 \\
\hline $\mathrm{MMB}$ & Memambetsu & 43.91 & 144.19 & 2440 \\
\hline PET & Paratunka & 52.97 & 158.25 & 1000 \\
\hline SIT & Sitka & 57.06 & 224.67 & 3100 \\
\hline $\mathrm{LZH}$ & Lanzhou & 36.1 & 103.84 & 5450 \\
\hline
\end{tabular}

the $Y$ component (E-W). The observed signals are similar to Pc3 geomagnetic pulsations. Typical Pc3 pulsations is a dayside/morning phenomenon, they last for $\sim 2-4$ hours, and are observed predominantly in the $X(\mathrm{~N}-\mathrm{S})$ component. In contrast to them, oscillations detected before the BSM explosions are short-lived, and are more evident in $Y$ component.

Figure 3 shows the result of time-frequency analysis (sonogram) of the signal $\mathrm{Y}$ component recorded at selected magnetic stations. These spectrograms confirm the appearance of magnetic field pulsations before the explosion. The dominant oscillation frequency is $\sim 25-35 \mathrm{mHz}$ (period is $\sim 0.5 \mathrm{~min}$ ). This period corresponds to typical resonant eigenfrequencies of the magnetospheric field lines at the latitudes under study.

More sensitive search-coil magnetometers at MGD and PET confirm the occurrence of weak oscillations with peak-to-peak amplitude $\sim 0.005 \mathrm{nT} / \mathrm{s}$ in the Pc3 band before the explosion (Figure 4).
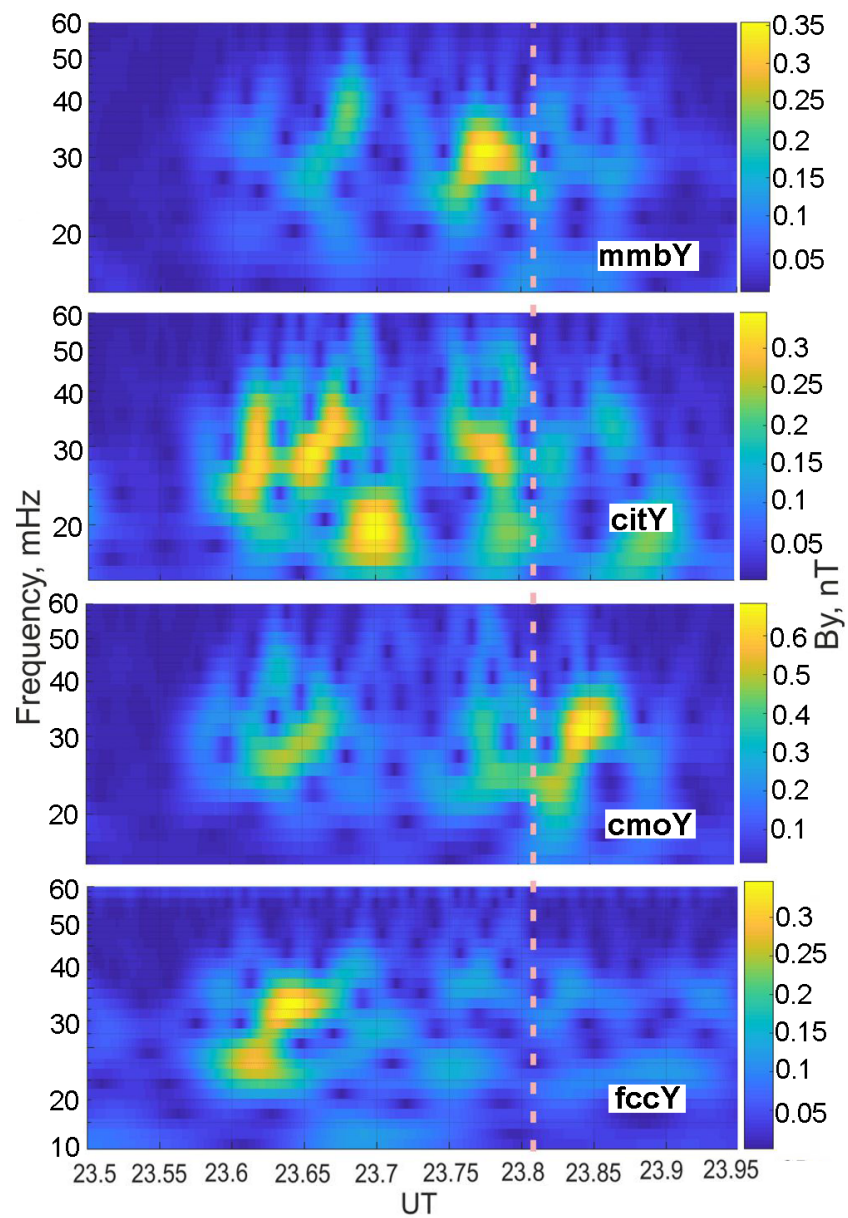

Figure 3. The result of time-frequency analysis of the signal for $\mathrm{Y}$ components of the magnetic field for MMB, SIT, CMO and FCC observatories. The red dashed line shows the moment of the explosion.

A clear wave packet appeared in $Y$ component $\sim 3$ min before the explosion and disappeared just after it.

At stations located far from the explosion site (about $5 \cdot 10^{3} \mathrm{~km}$ and father), e.g. Lanzhou (LZH) to the West and Ottawa (OTT) to the East, oscillations are practically undetectable. Taking into account the fact that according to NASA database https://cneos.jpl.nasa.gov/fireballs, the horizontal component of the BSM speed was 6.3 $\mathrm{km} / \mathrm{s}$, during the registration of geomagnetic variations the horizontal projection of its trajectory did not exceed $5 \cdot 10^{3} \mathrm{~km}$. Thus, the most intense quasiharmonic 30-s geomagnetic fluctuations have been observed at stations located inside the magnetic tube through which the meteoroid entered.

If one suggests that during the interaction of 

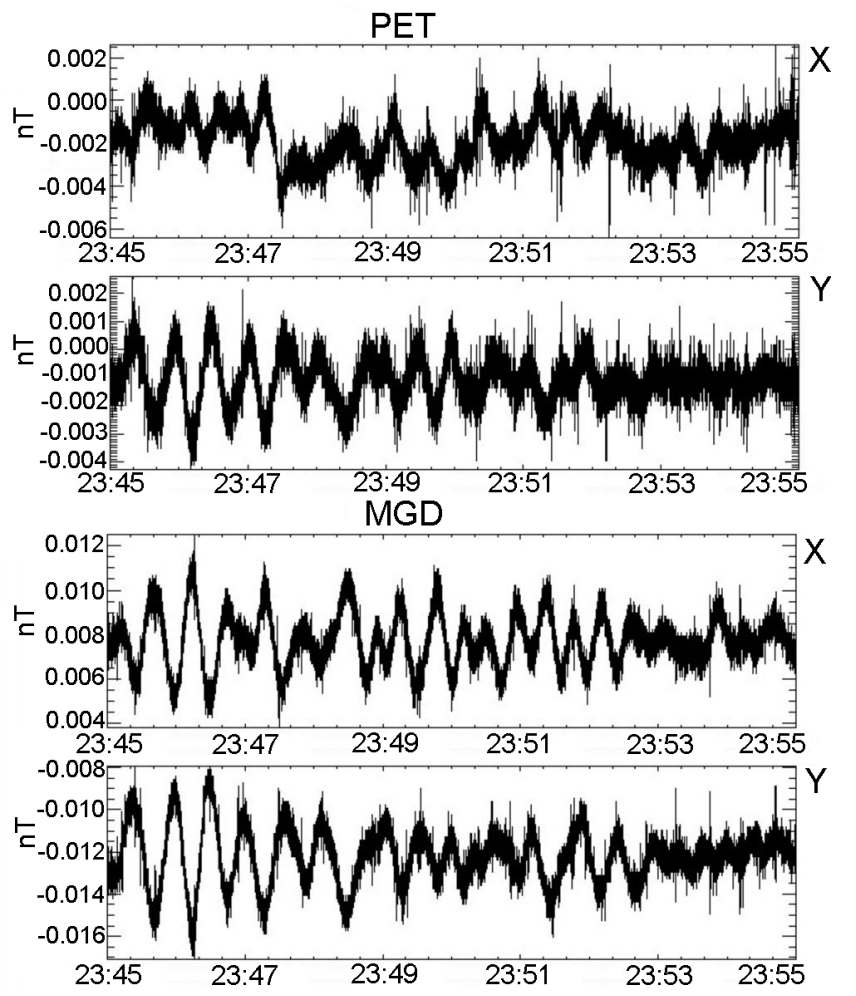

Figure 4. Variations of $\mathrm{X}$ and $\mathrm{Y}$ components of the magnetic field according to data of the Paratunka (PET) and Magadan (MGD) observatories on December 18, 2018.

the BSM with the magnetospheric plasma the field line oscillations are excited, then the occurrence of similar oscillation train is to be expected in magnetically conjugated point in the Southern hemisphere. To test this assumption, records of geomagnetic variations at Alice Springs $(\mathrm{ASP})$, Canberra (CNB), Cocos-Keeling Islands (CKI) and Macquarie Island (MCQ) in Australia, as well as Eyrewell $(\mathrm{EYR})$ in New Zealand, have been examined. The location of this stations is shown in Figure 5. Spectrograms of magnetic field oscillations from these stations are shown in Figure 6. It can be seen that small-amplitude oscillations similar to those found in Northern hemisphere can be seen only at two stations, CNB and EYR, conjugate to the explosion flux tube. Thus, the effect is localized also in the Southern hemisphere in the region magnetically conjugated with the meteoroid explosion site. Figure 7]shows the result of comparing of magnetic oscillations in the conjugate points of the two hemispheres.

\section{Discussion}

A magnetic effect of a meteor can be produced by the ionization along its trail and ionospheric electric current modified by this ionization. The sudden creation of extra ionization and conductivity is equivalent to the superposition of an additional current system whose magnetic field may be observable at the ground. The radar detectable ionization by meteors is usually produced between 80 and $110 \mathrm{~km}$. The theoretical order-of-magnitude estimation of a meteor magnetic effect can be made with a simple model as a vertical uniform cylinder with increased conductivity $\Sigma_{1}$ immersed into a uniform ionosphere with conductivity $\Sigma_{0}$ bounded by horizontal planes at heights $h$ and $h+d$, and background ionospheric current density $j_{o}$ [Chapman and Ashour, 1965. The total excess current $J$ flowing through the cylinder with diameter $a$ produces the magnetic response on the ground beneath the meteor trail which can be estimated as follows:

$$
J=2 j_{0} a d \frac{\Sigma_{1}-\Sigma_{0}}{\Sigma_{1}+\Sigma_{0}} \quad \Delta B=\frac{\mu_{0} J a}{2 h(h+d)} .
$$

For parameters $h=100 \mathrm{~km}, d=30 \mathrm{~km}, j_{o}=10^{-6}$ $\mathrm{A} / \mathrm{m}^{2}, \Sigma_{1} / \Sigma_{0}=3, a=30 \mathrm{~m}$, this estimate gives the expected magnetic effect $\Delta B \sim 2 \mathrm{nT}$. However, no geomagnetic response has been detected when the BSM was in the ionospheric E-layer. Probably, the lapse time when the BSM crossed the conductive E-layer, $<1$ s, was too short to excite prolonged oscillations.

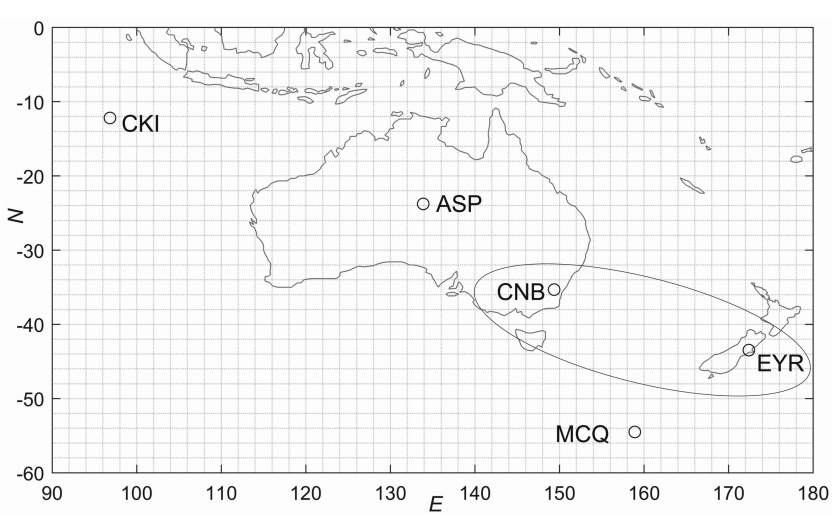

Figure 5. The position of the Alice Springs (ASP), Canberra (CNB), Cocos Islands (CKI) and Macquarie island (MCQ) observatories in Australia and Airwell (EYR) in New Zealand in the area magnetically coupled with BSM explosion of the southern hemisphere. 


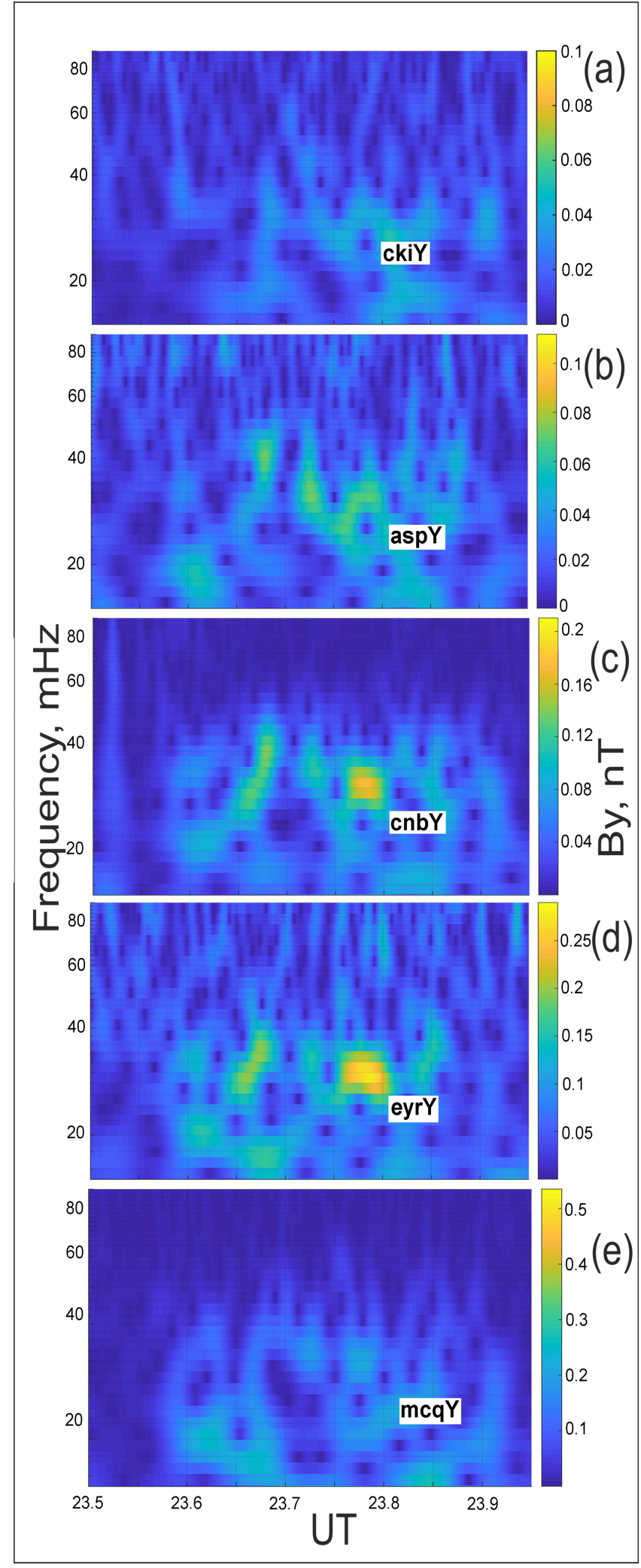

Figure 6. Spectrograms of magnetic field oscillations for CKI (a), ASP (b), CNB (c), EYR (d) and MCQ (e) stations. The red dashed line shows the moment of the explosion.

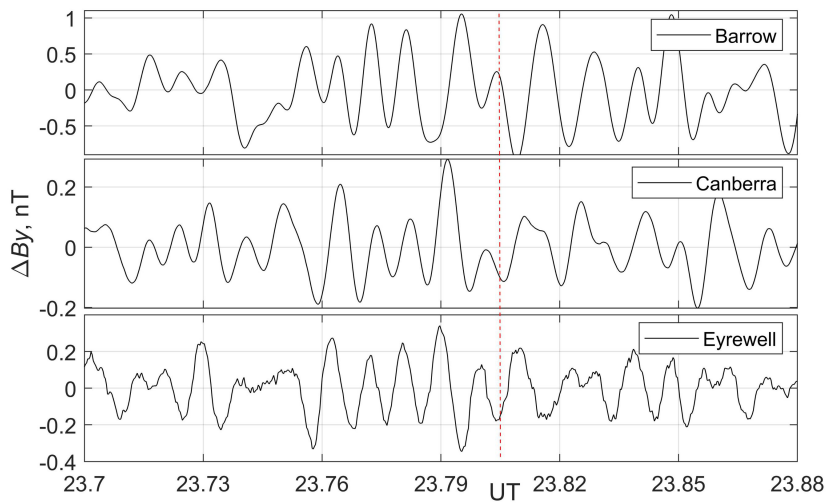

Figure 7. The result of comparing of magnetic oscillations for BRW, CNB and EYR stations in the magnetically conjugate regions of the two hemispheres.

Another electromagnetic effect which could cause the excitation of geomagnetic pulsations is associated with the movement of a conducting body with velocity $V_{0}$ through a magnetized plasma is the formation of the Alfven wings [Dobrowolny and Veltri, 1986. A front of field-aligned currents moves away from the body at an angle with geomagnetic field $\theta=\arctan \left(V_{0} / V_{A}\right)$, where $V_{A}$ is the Alfven velocity. The current flowing along an Alfven wing is $J=4\left[V_{0} \times B\right] a \Sigma_{A}$, where $a$ is the scale of a body. The factor $\Sigma_{A}=\left(\mu V_{A}\right)^{-1}$ is the Alfven wave conductance. This factor is larger inside the plasmasphere, so the Alfven wing generation is more efficient in this domain.

Chernogor 2018 interpreted the geomagnetic field perturbations observed before the Chelyabinsk Meteoroid impact as a result of magnetic field expulsion from the meteoroid trail. To achieve an agreement with ground observations, he had to assume that the magnetospheric magnetic field was completely expelled from an extended cavity with length $\sim 1.5 R_{E}$ and diameter $\sim 2 R_{E}$. A diamagnetic cavity of this size could only be formed if all the kinetic energy of the bolide was used to transfer the meteoroid substance to the plasma state. Such a situation is obviously impossible for BSM at a distance of several thousand kilometers from Earth. However, due to partial ablation of the meteorite, the diamagnetic effect could cause a local disturbance of the geomagnetic field and its propagation in the magnetic force tube.

We must take into account that the magnetosphere is often in a metastable state, when even 
a relatively weak external trigger can stimulate an internal instability and wave generation. As an example, one may recall the triggering by solar wind pressure pulses of magnetospheric long-lasting Pc1 emissions or global Pc5 waves [Pilipenko, 2013]. In line with this, a possibility of bolide flight to be an external trigger of resonant field line oscillations cannot be excluded. Although the presented evidences indicate that the recorded burst of oscillations in the frequency range around the eigenfrequency of magnetospheric field lines at a latitude under consideration are indeed triggered by a bolide transition through the inner magnetosphere, a possibility of accidental coincidence cannot be absolutely excluded. Nonetheless, keeping in mind a rare occurrence of such events, all associated effects must be examined.

\section{Conclusion}

The purpose of this study is to consider electromagnetic phenomena that could be caused by the fall of a large meteoroid over the Bering sea. It is surprising that despite a rather significant scale of the event, it did not attract much attention of the scientific community. There is a certain discrepancy in the explosion energy of this meteoroid, which is estimated from the optical data as $173 \mathrm{kT}$, and rather weak acoustic and electromagnetic effects that it caused. Unexpectedly, we found that the magnetic signal is recorded before the moment of explosion, so the signal may be generated in the inner magnetosphere during the passage of the meteoroid. The found excitation of magnetic pulsations $\sim 10$ minutes before the meteoroid explosion and an absence of electromagnetic signals directly associated with the explosion is unusual for such events. Our main purpose is to draw attention to this problem. Further research on these and other manifestations of the entry of a large space body into the Earth's ionosphere and magnetosphere are necessary. More complete set of observational effects will help to achieve an adequate understanding of the physical phenomena associated with meteorites, to improve methods for detecting and evaluating their characteristics, and to identify approaches to the problem of meteoroid danger prevention.
Acknowledgments. The authors are sincerely grateful to NASA fireball database (https://cneos.jpl.nasa.gov /fireballs), INTERMAGNET/!(https://www.intermagnet. org), PWING (http://www.isee.nagoya-u.ac.jp/ dimr/PWING) and IMS (http://www.cpdnp.jp/) teams for providing the measurement data. The help of $\mathrm{O}$. V. Kozyreva and useful comments of the reviewer are appreciated.

\section{References}

Adushkin, V. V., O. P. Popova, Yu. S. Rybnov, at al. (2004), Geophysical effects of the Vitim bolide September 24, 2002, Doklady Earth Sciences, 397, 685-688. (in Russian)

Bronshten, V. A. (1991), Electrical and electromagnetic phenomena associated with the meteor flight, Sol. Sys. Res., 25, 93-104.

Bronshten, V. A. (2002), Magnetic effect of the Tungus meteorite, Geomagn. Aeron., 42, 816-818.

Brown, P., R. Spalding, D. ReVelle, at al. (2002), The flux of small near-Earth objects colliding with the Earth, Nature, 420, 294-296, Crossref

Chapman, R., A. A. Ashour (1965), Meteor geomagnetic effects, Smithsonian Contributions to Astrophysics, 8 (7), 181-197, Crossref

Chernogor, L., N. Blaunstein (2013), Radiophysical and Geomagnetic Effects of Rocket Burn and Launch in the Near-the-Earth Environment, 542 pp. CRC Press, Boca Raton. Crossref

Chernogor, L. F. (2018), Effects during the Approach of the Chelyabinsk Meteoroid, Geomagn. Aeron., 58, 252-265, Crossref

Dobrowolny, M., P. Veltri (1986), Structure of Alfven wings associated with a conductor moving across a magnetoplasma, Astron. Astrophys., 167, 179185.

Ellyett, C. D., B. J. Fraser (1963), On the correlation of meteors with micropulsations, J. Geophys. Res., Vol. 68, Iss. 21, 5937-5945, Crossref

Gavrilov, B. G., J. I. Zetzer, I. M. Podgorny, et al. (2003), Plasma Jet Motion Across the Geomagnetic Field in the "North Star" Active Geophysical Experiment, Cosmic Researc, 41, 28-38, Crossref

Gordeev, E. I., S. N. Kulichkov, P. P. Firstov, et al. (2018), Infrasonic Waves and Assessment of the Explosion Energy of the Bering Sea Meteoroid on December 19, 2018, Dokl. Earth Sc., 489, 1436-1439, Crossref

Hawkins, G. S. (1958), A search for magnetic effects from meteors. Journal of Geophysical Research, J. Geophys. Res., 63(3), 467-475, Crossref

Ivanov, K. G. (1967), About the nature of the impact of the Tunguska fall on the upper atmosphere, the geomagnetic field and the glow of the night, Geomagn. 
Aeron., 7, No. 6, 1031-1035. (in Russian)

Ivanov, V. V., Y. A. Medvedev (1965), The magnetic effect and shock wave of a meteor, Soviet Astronomy, $8,890$.

Kalashnikov, A. G. (1949), On observations of magnetic effect of meteors by the induction method, Dokl. Akad. Sci. USSR, 66, 373-376.

Kalashnikov, A. G. (1952), On observations of magnetic effect of meteors by the induction method, Izvestia AN SSSR, Geofizika, No. 6, 7-20.

Negraru, P., G. Johnson (2019), Analysis of the infrasound signals from a bolide over the Bering Sea, CTBT Science and Technology 2019 Conference, T2.3-P005, Hofburg, Vienna, Austria.

Nemchinov, I. V, T. V. Loseva, V. G. Mukhin (1999), Estimation of the magnetic effect during the fall of the Tunguska meteoroid, Physical processes in the geospheres: their manifestations and interaction, $M$ : IDG RAS, 324-338,

Pilipenko, V. A. (2013), Trigger excitation of ULF waves in the Earth's magnetosphere, p. 318-328, GEOS, Moscow, Russia. (ISBN 978-5-89118641-5)

Popova, O. P., P. Jenniskens, V. V. Emelyanenko, et al. (2013), Chelyabinsk Airburst, Damage Assessment, Meteorite Recovery, and Characterization, Science, 342, 1069-1073, Crossref
Revelle, D. O. (1997), Historical Detection of Atmospheric Impacts by Large Bolides Using AcousticGravity Waves, Annals of the New York Academy of Sciences, 822, 284-302, Crossref

Savchenko, Yu. N. (1975), Geomagnetic Disturbances Caused by Shock Waves of Large Meteoric Bodies, Geomagn. Aeron., 15, No. 6, 1047-1053.

Savchenko, Yu. N. (1976), Geomagnetic Disturbances Caused by Shock Waves of Large Meteoric Bodies. II, Geomagn. Aeron., 16, No. 6, 518-525.

Shiokawa, K., Y. Katoh, Y. Hamaguchi, et al. (2017), Ground-based instruments of the PWING project to investigate dynamics of the inner magnetosphere at subauroral latitudes as a part of the ERG-ground coordinated observation network, Earth Planets Space, 69, 160, Crossref

Zetser, Y. I., B. G. Gavrilov, V. A. Zhmailo, et al. (2004), Geomagnetic Effects from Expanding Plasma Formation of a High-Altitude Nuclear Explosion, Combustion, Explosion, and Shock Waves, 40, 638-648, Crossref

\section{Corresponding author:}

V. A. Pilipenko, Geophysical Center RAS, 3 Molodezhnaya St., 119296 Moscow, Russia. (pilipenko_va@mail.ru) 